\title{
QUARTIC STRUCTURES ON SPHERES
}

\author{
SAMUEL I. GOLDBERG
}

\section{Introduction}

A $C^{\infty}$ tensor field $f$ of type $(1,1)$ on a connected $C^{\infty}$ manifold $P$ is said to define a polynomial structure of degree $d$ if $d$ is the smallest integer for which the powers $I, f, \cdots, f^{d}$ are dependent, and $f$ has constant rank on $P$, where $I$ is the identity transformation field. An almost complex manifold is a polynomial structure of degree 2 . In the odd dimensional case, the almost contact manifolds provide examples of polynomial structures of degree 3. More generally, a globally framed $f$-manifold is a polynomial structure of degree 3 . These are almost product spaces. In addition, almost product spaces are a source of further examples of polynomial structures [8].

The affine spaces $R^{2 n}$ and $R^{2 n-1}$ may be endowed with almost complex and almost contact structures, respectively, so these give the simplest examples of the manifolds considered the former having rank $2 n$ and the latter rank $2 n-2$. On the other hand, an odd dimensional sphere $S^{2 n-1}$ carries an almost contact structure, so it is a polynomial manifold which is globally framed. However, the even dimensional spheres are not almost complex except in dimensions 2 and 6 , and whereas the contact structure on $S^{2 n-1}$ is "integrable", it is not even known whether $S^{6}$ can be given an almost complex structure which comes from a complex structure.

In a previous work [6], polynomial structures $f$ of degree 4 were introduced and examples of them provided. These were of two types, namely,

$$
f^{4}+f^{2}=0, \quad\left(f^{2}+I\right)^{2}=0,
$$

the first one having rank $2 n-1$ and the second maximal rank $2 n$. Moreover, the former is globally framed and the latter is not. We show below that, except for a set of measure zero, the even dimensional spheres may be endowed with a quartic structure $f$, depending on a parameter $\lambda$, that is,

$$
\left(f^{2}+\lambda^{2} I\right)\left(f^{2}+I\right)=0, \quad 0<|\lambda| \leq 1,
$$

and

Communicated by K. Yano, March 4, 1970. Research partially supported by the National Science Foundation. 


$$
f^{3}+f=0
$$

on the set of points for which $\lambda=0$. On the other hand, each of the connected components $S^{2 n} \backslash\left\{p \in S^{2 n} \mid \lambda(p)=0\right\}$ may be characterized as a complete totally umbilical noninvariant hypersurface of a cosymplectic manifold of constant curvature.

It is these structures which we are preoccupied with below, that is, with even or odd dimensional manifolds carrying a quartic structure of arbitrary rank.

\section{Noninvariant hypersurfaces}

We motivate our continuing study of polynomial structures by considering a $2 n$-dimensional manifold $P$ imbedded as a hypersurface in an almost contact manifold $M(\varphi, E, \eta), i: P \rightarrow M$ being the imbedding, $\varphi$ the fundamental affine collineation of $M, E$ its fundamental vector field and $\eta$ the contact form. It is assumed that $E$ is nowhere tangent to the hypersurface $i(P)$. Denoting by $i_{*}$ the induced tangent map of $i$, we therefore have

$$
\varphi i_{*} X=i_{*} J X+\alpha(X) E, \quad \varphi E=0,
$$

where $J$ is an almost complex structure on $P$ and $\alpha(J X)=\eta\left(i_{*} X\right)$. Thus $P$ admits an almost complex structure $J$ and a 1-form $\alpha$ whose vanishing at $p \in P$ means that the tangent hyperplane at $p$ of the hypersurface is invariant by $\varphi_{p}$. If $\alpha \neq 0$, the submanifold $i(P)$ is called a noninvariant hypersurface [3].

It is well-known that a metric $G$ may be introduced on $M$ with the properties

$$
G(\varphi x, y)=-G(x, \varphi y)
$$

where $x$ and $y$ are vector fields on $M$, that is, $\varphi$ is skew symmetric with respect to $G$, and

$$
\eta=G(E, \cdot) .
$$

Let $N$ be the unit normal to $i(P)$ with respect to $G$. Then we may write

$$
\varphi i_{*} X=i_{*} f X+\alpha^{\prime}(X) N
$$

for some $(1,1)$ tensor field $f$ and 1 -form $\alpha^{\prime}$ on $P$. Moreover, since $\varphi N$ is orthogonal to $N$ with respect to $G$, it is tangent to $i(P)$ and so can be expressed as

$$
\varphi N=-i_{*} U
$$

for some vector field $U$ on $P$. There is clearly a relation between $f, J, \alpha$ and $\alpha^{\prime}$. Indeed, since $E$ is nowhere tangent to $P$,

$$
E=i_{*} V+\lambda N, \quad \lambda \neq 0,
$$


for some vector field $V$ and scalar field $\lambda$ on $P$. Clearly, $|\lambda| \leq 1$.

From (2.2) and (2.5), $\eta(N)=\lambda$. Comparing (2.1) with (2.3) yields, by virtue of (2.5),

$$
f=J+\alpha \otimes V, \quad \alpha^{\prime}=\lambda \alpha .
$$

If we put $\beta=i^{*} \eta$ and $(C \alpha)(X)=\alpha(J X)$, then $\beta=C \alpha$. Summarizing, we have proved (cf. [1])

Theorem 1. Let $P(J, \alpha)$ be a noninvariant hypersurface of the almost contact metric manifold $M(\varphi, \eta, G)$. Then there exist tensor fields $f, U, V, \alpha, \alpha^{\prime}, \beta$ and $\lambda, 0<|\lambda| \leq 1$, on $P$ defined by

(i) $\varphi i_{*} X=i_{*} f X+\alpha^{\prime}(X) N, \quad \alpha^{\prime}=\lambda \alpha$,

(ii) $\varphi N=-i_{*} U$,

(iii) $E=i_{*} V+\lambda N$,

(iv) $\beta=C \alpha=i^{*} \eta$,

(v) $\lambda=\eta(N)$.

The vector fields $U$ and $V$ vanish at those points on $P$ for which $\lambda= \pm 1$, and at these points $f$ coincides with $J$.

Noting that $\varphi^{2}=-I+\eta \otimes E$, Theorem 1 yields

Proposition 2. The structure tensors on the noninvariant hypersurface $P$ satisfy

(i) $f^{2}=-I+\alpha^{\prime} \otimes U+\beta \otimes V$,

(ii) $\alpha^{\prime} \circ f=\lambda \beta, \quad \beta \circ f=-\lambda \alpha^{\prime}$,

(iii) $f U=-\lambda V, \quad f V=\lambda U$,

(iv) $\alpha^{\prime}(U)=1-\lambda^{2}, \quad \alpha^{\prime}(V)=0$,

(v) $\beta(U)=0, \quad \beta(V)=1-\lambda^{2}$.

Corollary 1. $P(f, U, V, \alpha, \beta, \lambda)$ is a quartic structure of maximal rank.

In fact,

$$
f^{4}+\left(1+\lambda^{2}\right) f^{2}+\lambda^{2} I=0
$$

that is,

$$
\left(f^{2}+\lambda^{2} I\right)\left(f^{2}+I\right)=0 .
$$

If $f X=0$, then $X=\alpha^{\prime}(X) U+\beta(X) V$. Hence $0=\alpha^{\prime}(X) f^{2} U+\beta(X) f^{2} V=$ $-\lambda^{2}\left(\alpha^{\prime}(X) U+\beta(X) V\right)$, so $\lambda$ being nowhere zero, $X$ must vanish.

In the sequel, unless explicitly mentioned to the contrary, we assume that $\lambda \neq \pm 1$ and, in this case, we denote by $P^{\prime}$ the manifold defined by $\{p \in P \mid 0<\lambda(p)<1\}$. Geometrically this means that $E$ and $N$ are distinct vector fields in the sense that $E_{i(p)} \neq N_{i(p)}$ for all $p \in P^{\prime}$. This means that the EulerPoincaré characteristic $\chi\left(P^{\prime}\right)$ of $P^{\prime}$ is zero since $U$ (and $V$ ) are nonsingular vector fields on $P^{\prime}$. Topologically, this is the case for the odd dimensional spheres, but $\chi\left(S^{2 n}\right) \neq 0$. Note however that there are points on $S^{2 n}-\left\{p \in S^{2 n} \mid \lambda(p)\right.$ 
$=0\}$, considered as a noninvariant hypersurface, for which $\lambda= \pm 1$.

If we put $\tilde{\alpha}=\left(1-\lambda^{2}\right)^{-1} \alpha^{\prime}$ and $\tilde{\beta}=\left(1-\lambda^{2}\right)^{-1} \beta$, then $P^{\prime}$ is pseudo-globally framed, that is, $\tilde{\alpha}(U)=1, \tilde{\alpha}(V)=0, \tilde{\beta}(U)=0, \tilde{\beta}(V)=1$ and

$$
f^{2}=-I+\rho(\tilde{\alpha} \otimes U+\tilde{\beta} \otimes V), \quad \rho=1-\lambda^{2} .
$$

Corollary 2. The noninvariant hypersurface $P^{\prime}$ is pseudo-globally framed. Moreover,

$$
\tilde{\alpha} \circ f=\lambda \tilde{\beta}, \quad \tilde{\beta} \circ f=-\lambda \tilde{\alpha},
$$

and

$$
f U=-\lambda V, \quad f V=\lambda U
$$

If we put

$$
f_{1}=f-\lambda \tilde{\beta} \otimes U
$$

and compute its square, we get $f_{1}^{2} X=-X+\tilde{\alpha}(X) U+\tilde{\beta}(X) V$. Moreover, $f_{1} U=-\lambda V$ and $f_{1} V=0$. Hence $f_{1}^{3} X=-f_{1} X-\lambda \tilde{\alpha}(X) V$, from which $f_{1}^{4} X=$ $-f_{1}^{2} X$. If $f_{1} X=0$, then $X=\tilde{\alpha}(X) U+\tilde{\beta}(X) V$. Applying $f_{1}$ again gives $0=$ $-\lambda \tilde{\alpha}(X) V$, so $\tilde{\alpha}(X)=0$ since $\lambda$ and $V$ are nowhere zero. Thus, rank $f_{1}=2 n-1$.

An even dimensional $C^{\infty}$ manifold $P$ is said to be globally framed if there exist a linear transformation field $f$, global vector fields $E_{a}$ and 1 -forms $\eta^{a}, a=$ $1, \cdots, 2 \nu$, satis fying

$$
\begin{gathered}
\eta^{a}\left(E_{b}\right)=\delta_{b}^{a}, \\
f^{2}=-I+\eta^{a} \otimes E_{a},
\end{gathered}
$$

the summation convention being employed here and in the sequel.

Theorem 3. The noninvariant hypersurface $P^{\prime}$ carries a globally framed quartic structure $\left(f_{1}, U, V, \tilde{\alpha}, \tilde{\beta}\right)$ of rank $2 n-1$, where $f_{1}=f-\lambda \tilde{\beta} \otimes U$, that is,

(i) $f_{1}^{2}=-I+\tilde{\alpha} \otimes U+\tilde{\beta} \otimes V$,

(ii) $\tilde{\alpha} \circ f_{1}=0, \quad \tilde{\beta} \circ f_{1}=-\lambda \tilde{\alpha}$,

(iii) $f_{1} U=-\lambda V, \quad f_{1} V=0$,

(iv) $\tilde{\alpha}(U)=1, \quad \tilde{\alpha}(V)=0$,

(v) $\tilde{\beta}(U)=0, \quad \tilde{\beta}(V)=1$, and

$$
f_{1}^{4}+f_{1}^{2}=0 .
$$

Corollary. The noninvariant hypersurface $P^{\prime}(J, \alpha, g)$ of the almost contact metric manifold $M(\varphi, \eta, G)$ may be endowed with a quartic structure of maximal rank $2 n$ given by the $(1,1)$ tensor field 


$$
J-\lambda\left(\alpha \otimes J U+\frac{1}{1-\lambda^{2}} C \alpha \otimes U\right)
$$

where $U$ is the vector field on $P^{\prime}$ (of length 1) given by $\varphi N=-i_{*} U$ and the function $\lambda$ is defined by $E=\lambda\left(N-i_{*} J U\right), \eta=G(E, \cdot)$.

If we put $f_{2}=f_{1}+\lambda \tilde{\alpha} \otimes V$, then $f_{2} U=0$ and $f_{2} V=0$. Moreover, $f_{2}^{2} X=$ $-X+\tilde{\alpha}(X) U+\tilde{\beta}(X) V$. Applying $f_{2}$ again gives $f_{2}^{3} X=-f_{2} X$.

Theorem 4. The noninvariant hypersurface $P^{\prime}$ carries a globally framed $f-$ structure $\left(f_{2}, U, V, \alpha, \beta\right)$ of rank $2 n-2$, that is,

(i) $f_{2}^{2}=-I+\tilde{\alpha} \otimes U+\tilde{\beta} \otimes V$,

(ii) $\tilde{\alpha} \circ f_{2}=0, \quad \tilde{\beta} \circ f_{2}=0$,

(iii) $f_{2} U=0, \quad f_{2} V=0$,

(iv) $\tilde{\alpha}(U)=1, \quad \tilde{\alpha}(V)=0$,

(v) $\tilde{\beta}(U)=0, \quad \tilde{\beta}(V)=1$,

where

$$
f_{2}=f-\lambda(\tilde{\beta} \otimes U-\tilde{\alpha} \otimes V) .
$$

Corollary. The noninvariant hypersurface $P^{\prime}(J, \alpha, g)$ of the almost contact metric manifold $M(\varphi, \eta, G)$ may be endowed with an $f$-structure of rank $2 n-2$ defined by the $(1,1)$ tensor field

$$
J-\frac{\lambda}{1-\lambda^{2}}(C \alpha \otimes U+\alpha \otimes J U)
$$

where $U$ is the vector field on $P$ (of length 1) given by $\varphi N=-i_{*} U$ and the scalar field $\lambda$ is given by $E=\lambda\left(N-i_{*} J U\right), \eta=G(E, \cdot)$.

\section{Symplectic quartic structures}

Since the collineation $\varphi$ is skew symmetric with respect to the metric $G$, the field $f$ has this property with respect to the induced metric. For, $g(f X, Y)=$ $G\left(i_{*} f X, i_{*} Y\right)=G\left(\varphi i_{*} X-\alpha^{\prime}(X) N, i_{*} Y\right)=-G\left(i_{*} X, \varphi i_{*} Y\right)=-G\left(i_{*} X, i_{*} f Y\right)$ $=-g(X, f Y)$. We put

$$
F(X, Y)=g(f X, Y)
$$

and call $F$ the fundamental form of the noninvariant hypersurface $P(f, U, V, g)$. Clearly,

$$
F=i^{*} \Phi
$$

where $\Phi$ is the fundamental form of the ambient space.

From (2.3)-(2.5), 


$$
\alpha^{\prime}=g(U, \cdot), \quad \beta=g(V, \cdot) .
$$

Moreover, from formula (iv) of Proposition 2, $U$ and $V$ are orthogonal, that is, $g(U, V)=0$. If we put $\bar{g}=\left(1-\lambda^{2}\right)^{-1} g$ on $P^{\prime}$, then $U$ and $V$ form an orthonormal pair with respect to the metric $\bar{g}$. Setting $F_{2}=g\left(f_{2} X, Y\right)$, we get

$$
F_{2}=F+2 \lambda\left(1-\lambda^{2}\right) \tilde{\alpha} \wedge \tilde{\beta} .
$$

Assume now that $M$ is normal and $\Phi$ is closed; for example, assume that $M$ is a cosymplectic manifold. Then, $F$ is closed, so by Corollary 1 to Proposition 2, the 'quartic' structure on $P$ is symplectic, that is, $F$ is closed and of maximal rank. (The ambient space cannot be a contact metric manifold since $P$ is a noninvariant hypersurface [3].) Observe that $F(X, Y)=g(f X, Y)=$ $g(J X, Y)+\alpha(X) \beta(Y)$, so $F(X, Y)=\frac{1}{2}[g(J X, Y)-g(X, J Y)]+(\alpha \wedge \beta)(X, Y)$. Since $\alpha \neq 0,(J, g)$ is not an hermitian structure on $P$. However, $\left(J, g^{*}\right)$ is hermitian where $g^{*}=g+\alpha \otimes \alpha$. Indeed, $g^{*}(J X, Y)=g(J X, Y)+\beta(X) \alpha(Y)$ $=F(X, Y)-\alpha(X) \beta(Y)+\alpha(Y) \beta(X)=F(X, Y)-2(\alpha \wedge \beta)(X, Y)$. Putting

$$
\Omega^{*}(X, Y)=g^{*}(J X, Y),
$$

it is easily seen that

$$
F=\Omega^{*}+2 \alpha \wedge \beta
$$

Since $f$ is of maximal rank and $i$ is a regular map, the symmetric tensor

$$
\gamma=g-\beta \otimes \beta
$$

defines a Riemannian metric, in fact, an hermitian metric with respect to $J$. Since $\Phi$ is closed, $\gamma$ is an almost Kaehler metric and $F$ is the fundamental form of the almost Kaehler manifold $P(J, \gamma)$. M being normal, $P(J, \gamma)$ is a Kaehler manifold [3].

Denote by $K$ the ring of real-valued functions on $P$. To each vector field $X$ on $P$, we assign the 1 -form $\xi$ defined by

$$
\xi=\iota(X) F
$$

where $\iota$ is the interior product operator given by

$$
[\iota(X) \theta]\left(X_{1}, \cdots, X_{p-1}\right)=p \theta\left(X, X_{1}, \cdots, X_{p-1}\right),
$$

$\theta$ being a $p$-form, $p \geq 1$, and $\iota(X) k=0, k \in K$. We therefore have an isomorphism $\mu$ of the $K$-module of vector fields on $P$ onto the $K$-module of 1 forms on $P$ defined by $\mu(X)=\xi$. This isomorphism may be naturally extended to an isomorphism, again denoted by $\mu$, of the $K$-module of skew symmetric contravariant tensors of order $p$ with the $K$-module of $p$-forms. 
Following P. Libermann (see [7]) an operator $\widetilde{*}$ analogous to the Hodge star operator is defined as follows:

$$
\widetilde{*}=\iota\left(\mu^{-1} \theta\right) \frac{F^{n}}{n !} .
$$

If $\theta$ is a $p$-form, then $\widetilde{*} \theta$ is a $(2 n-p)$-form and

$$
\widetilde{*}^{2} \theta=\theta \text {. }
$$

We may also define the operator $L=\epsilon(F)$, the exterior product by $F$, on the symplectic manifold $P(f, g)$. It clearly coincides with the corresponding operator of Hodge-Weil on Kaehler manifolds. We shall see below that its "dual" $\tilde{\Lambda}$ with respect to $\widetilde{*}$ coincides with its dual with respect to $*$. In fact,

$$
\tilde{\Lambda}=\widetilde{*}^{-1} L \widetilde{*}=\widetilde{*} L \widetilde{*}
$$

Thus

$$
\tilde{\Lambda} \theta=\iota\left(\mu^{-1} F\right) \theta, \quad p \geq 2 .
$$

Analogous to the codifferential operator $\delta$, the symplectic codifferential operator $\tilde{\delta}$ is defined by

$$
\tilde{\delta}=\widetilde{*}^{-1} d \widetilde{*}=\widetilde{*} d \widetilde{*}
$$

Clearly

$$
\tilde{\delta}^{2}=0
$$

We relate the operators $\tilde{*}, \tilde{\Lambda}$ and $\tilde{\delta}$ on the symplectic manifold $P$ to the corresponding operators of Hodge-Weil on the underlying almost hermitian structure $(J, \gamma)$. Since $F(X, Y)=\gamma(J X, Y)$, we obtain

$$
\frac{F^{n}}{n !}=(-1)^{n(n-1) / 2} * 1,
$$

where $* 1$ is the volume element of $\gamma$. Clearly,

$$
*^{2}=(-1)^{p}
$$

on $p$-forms.

The operator $C$ previously applied to $\alpha$ may be extended to a mapping, again denoted by $C$, on $p$-forms $\theta$ as follows:

$$
C \theta\left(X_{1}, \cdots, X_{p}\right)=\theta\left(J X_{1}, \cdots, J X_{p}\right) .
$$


It has the obvious property

$$
C^{2} \theta=(-1)^{p} \theta
$$

Moreover, it commutes with $*$ and $L$. From the definition of $\widetilde{*}$, we obtain

$$
\widetilde{*}=(-1)^{n(n-1) / 2} * C^{-1} \text {. }
$$

Hence

$$
\tilde{\Lambda} \theta=\widetilde{*}^{-1} L \approx \theta=C *^{-1} L * C^{-1} \theta=C \Lambda C^{-1} \theta=C C^{-1} \Lambda \theta=\Lambda \theta,
$$

so the operators $\Lambda$ and $\tilde{\Lambda}$ coincide.

From (3.2), we get

$$
\tilde{\delta}=C \delta C^{-1} .
$$

For, $\tilde{\delta} \theta=C *^{-1} d * C^{-1} \theta=C \delta C^{-1} \theta$. Hence, from the formula

$$
d \Lambda-\Lambda d=-C \delta C^{-1}
$$

valid for almost hermitian manifolds, the purely symplectic relations

$$
d \Lambda-\Lambda d=-\tilde{\delta}
$$

and

$$
\tilde{\delta} L-L \tilde{\delta}=-d
$$

are obtained.

Finally, from (3.3) and (3.4),

$$
\int_{P} \tilde{\delta} \theta \widetilde{*} 1=0
$$

if $P$ is compact.

If $P^{\prime}$ is compact, its topology can therefore be studied from the symplectic point of view $(f, g)$ in comparison with the symplectic point of view $(J, \gamma)$ of Hodge-Weil, the interesting thing being that $F$ is the fundamental from of both structures.

\section{Hypersurfaces of cosymplectic spaces}

Denote by $\nabla$ the Riemannian connection of $M(\varphi, \eta, G)$ and by $D$ the induced connection on the hypersurface $P$. Then, the equations of Gauss and Weingarten are 


$$
\left(D_{X} i_{*}\right) Y=h(X, Y) N
$$

and

$$
D_{X} N \equiv \nabla_{i * X} N=-i_{*} H X
$$

respectively, where $h$ and $H$ are the second fundamental tensors (of types $(0,2)$ and $(1,1)$, respectively) of $P$ with respect to the normal vector field $N$. The tensor $h$ is symmetric and $h(X, Y)=g(H X, Y)$.

Covariant differentiation of (2.3) along $P$ yields

$$
\begin{aligned}
& \left(\nabla_{i * Y} \varphi\right) i_{*} X-h(X, Y) i_{*} U+i_{*} f D_{Y} X+\alpha^{\prime}\left(D_{Y} X\right) N \\
& =h(f X, Y) N+i_{*}\left(D_{Y} f\right) X+i_{*} f D_{Y} X+\left(D_{Y} \alpha^{\prime}\right)(X) N \\
& \quad+\alpha^{\prime}\left(D_{Y} X\right) N-\alpha^{\prime}(X) i_{*} H Y .
\end{aligned}
$$

Covariant differentiation of (2.4) along $P$ gives

$$
\left(\nabla_{i * X} \varphi\right) N-\varphi i_{*} H X=-h(X, U) N-i_{*} D_{X} U,
$$

from which follows

$$
\left(\nabla_{i_{*} X} \varphi\right) N-i_{*} f H X-\alpha^{\prime}(H X) N=-h(X, U) N-i_{*} D_{X} U .
$$

Differentiating (2.5) gives rise to

$$
\nabla_{i_{*} X} E=h(X, V) N+i_{*} D_{X} V+(X \lambda) N-\lambda i_{*} H X .
$$

Similarly we have, from $\beta=i^{*} \eta$,

$$
\left(D_{X} \beta\right)(Y)+\beta\left(D_{X} Y\right)=\left(\nabla_{i_{*} X} \eta\right)\left(i_{*} X\right)+h(X, Y) \eta(N)+\beta\left(D_{X} Y\right),
$$

and, from $\lambda=\eta(N)$,

$$
X \lambda=\left(\nabla_{i_{*} X} \eta\right)(N)-\beta(H X)
$$

If the ambient space is cosymplectic, then

$$
\nabla \varphi=0, \quad \nabla \eta=0, \quad \nabla E=0
$$

(see [3]). Thus

$$
\begin{aligned}
& \left(D_{X} f\right) Y=\alpha^{\prime}(Y) H X-h(X, Y) U, \\
& \left(D_{X} \alpha^{\prime}\right)(Y)=-h(X, f Y), \quad\left(D_{X} \beta\right)(Y)=\lambda h(X, Y), \\
& D_{X} U=f H X, \quad D_{X} V=\lambda H X, \\
& h(X, U)=\alpha^{\prime}(H X), \quad h(X, V)=\beta(H X), \\
& X \lambda=-\beta(H X) .
\end{aligned}
$$


Observe that $\beta$ is closed.

The manifold $P^{\prime}$ is said to be totally flat if its structure tensors are parallel fields with respect to the Riemannian connection.

$P^{\prime}$ is called normal if its induced globally framed $f$-structure is normal [4].

Proposition 5. If the ambient space of the symplectic quartic manifold $P$ is cosymplectic and $h=\mu \alpha \otimes \alpha$, then $f$ is covariant constant and $\lambda=$ const. On the other hand, if $P$ is totally umbilical, then $V$ is a conformal vector field. If $H$ commutes with $f$, then $U$ is a Killing vector field, and conversely.

Although $f$ has vanishing covariant derivative, $(f, g)$ is not normal (see $\S 6$ ). However, we do have

Corollary 1. If $P$ is totally geodesic and the ambient space is cosymplectic, then $P$ is totally flat and normal.

If $P$ satisfies the conditions in Corollary 1 , then $D J=0$ since $J=f-\alpha \otimes V$. Hence $\left(J, g^{*}\right)$, as well as $(J, \gamma)$, is a Kaehler structure on $P$.

Corollary 2. The hermitian structure $\left(J, g^{*}\right)$ on the totally geodesic hypersurface $P$ of the cosymplectic manifold $M$ is Kaehlerian.

Corollary 3. If the hypersurface $(P, g)$ is totally umbilical, that is, if $h=\sigma g$ (with respect to $N)$ and $(M, G)$ is of constant curvature, then for $n>1,(P, g)$ is of constant nonnegative curvature $\sigma^{2}$.

Proof. Indeed, $\nabla_{i_{*} X} i_{*} Y=i_{*} D_{X} Y+\sigma g(X, Y) N$, so

$$
\begin{gathered}
\nabla_{i_{*} Z} \nabla_{i_{*} X} i_{*} Y=i_{*} D_{Z} D_{X} Y+\sigma\left\{g\left(Z, D_{X} Y\right)+g\left(D_{Z} X, Y\right)+g\left(X, D_{Z} Y\right)\right. \\
+Z \sigma \cdot g(X, Y)\} N-\sigma^{2} g(X, Y) i_{*} Z
\end{gathered}
$$

Denoting by $\tilde{R}$ and $R$ the curvature tensors of the metrics $G$ and $g$, respectively, we get

$$
\begin{aligned}
\tilde{R}\left(i_{*} Z, i_{*} X\right) i_{*} Y= & i_{*}\left\{R(Z, X) Y-\sigma^{2}[g(X, Y) Z-g(Z, Y) X]\right\} \\
& +\{Z \sigma \cdot g(X, Y)-X \sigma \cdot g(Z, Y)\} N
\end{aligned}
$$

Thus, $\tilde{R}$ being zero,

$$
R(Z, X) Y=\sigma^{2}\{g(X, Y) Z-g(Z, Y) X\}
$$

and $Z \sigma \cdot g(X, Y)=X \sigma \cdot g(Z, Y)$, each of these relations implying $\sigma$ is constant on $M$.

Under the conditions of Corollary 3, equations (4.1) become

$$
\begin{aligned}
& \left(D_{X} f\right) Y=\sigma\left[\alpha^{\prime}(Y) X-g(X, Y) U\right], \\
& \left(D_{X} \alpha^{\prime}\right)(Y)=\sigma F(X, Y), \quad\left(D_{X} \beta\right)(Y)=\lambda \sigma g(X, Y), \\
& D_{X} U=\sigma f X, \quad D_{X} V=\sigma \lambda X, \\
& X \lambda=-\sigma \beta(X) .
\end{aligned}
$$


Observe that $F=-D \alpha^{\prime}$, so $F$ is an exact 2-form, that is, $F$ does not give rise to a nontrivial cohomology class. Moreover, the second and third statements of Proposition 5 clearly hold. It may also be checked that $d \alpha$ is of bidegree $(1,1)$ with respect to $J=f-\alpha \otimes V$.

In [3] it was shown that a noninvariant hypersurface of a cosymplectic manifold is a complex manifold. Thus $P(J, g)$, as well as $P^{\prime}(J, g)$, is endowed with the integrable almost complex structure of formula (2.1).

The even dimensional sphere: We regard $E^{2 n+1}$ as a cosymplectic space and let $\bar{S}^{2 n}$ be the unit sphere in $E^{2 n+1}$ minus the set of points (the equator) on which $\lambda=0$ (see also [1]). Then, since $S^{2 n}$ is a totally umbilical hypersurface of $E^{2 n+1}$ with $\sigma=-1$, equations (4.2) become

$$
\begin{aligned}
& \left(D_{X} f\right) Y=g(X, Y) U-\alpha^{\prime}(Y) X, \\
& \left(D_{X} \alpha^{\prime}\right)(Y)=-F(X, Y), \quad\left(D_{X} \beta\right)(Y)=-\lambda g(X, Y), \\
& D_{X} U=-f X, \quad D_{X} V=-\lambda X, \\
& X \lambda=\beta(X) .
\end{aligned}
$$

Thus, for every $n, \bar{S}^{2 n}$ (as well as $\left(S^{2 n}\right)^{\prime}=S^{2 n} \backslash\left\{p \in S^{2 n} \mid \lambda(p)=0,+1,-1\right\}$ ) carries the integrable almost complex structure of formula (2.1). (Observe that none of the points on $S^{2 n}$ for which $\lambda=0$ can be a zero of the closed conformal vector field $V$; see $[10$, p. 170].)

Corollary 4. If the hypersurface $(P, g)$ is totally geodesic and its ambient space $(M, G)$ is of constant curvature, then $(P, g)$ and $(P, \gamma)($ as well as $(M, G))$ are locally flat.

Proof. Let $\bar{D}$ denote the Riemannian connection of $\gamma$. Then, by the definition of the Riemannian connection and the formulas $D J=0$ and $\left(D_{X} \alpha\right)(Y)=$ $-h(X, J Y)($ see $[3])$, we get

$$
\begin{aligned}
\gamma\left(\bar{D}_{X} Y, Z\right) & =\gamma\left(D_{X} Y, Z\right)+h(X, Y) \beta(Z) \\
& =\gamma\left(D_{X} Y, Z\right)-h(X, Y) \gamma(J U, Z)
\end{aligned}
$$

so that

$$
\bar{D}_{X} Y=D_{X} Y-h(X, Y) J U=D_{X} Y,
$$

since $(P, \gamma)$ is a totally geodesic hypersurface [6].

Again, since $h=0$, the Gauss equation (with respect to $N$ ) is

$$
\nabla_{i_{*} X} i_{*} Y=i_{*} D_{X} Y=i_{*} \bar{D}_{X} Y \text {. }
$$

Thus, denoting the Riemannian curvature tensor of $(P, \gamma)$ by $\bar{R}$,

$$
\tilde{R}\left(i_{*} X, i_{*} Y\right) i_{*} Z=\left[\nabla_{i_{*} X}, \nabla_{i_{*} Y}\right] i_{*} Z-\nabla_{\left[i_{*} X, i_{*} Y\right]} i_{*} Z=i_{*} \bar{R}(X, Y) Z,
$$


from which $\gamma$ is a locally flat metric since $\tilde{R}=0$ and $i$ is a regular map.

Corollary 5. Under the conditions of Corollary 3 , if $P(J, g)$ is an invariant hypersurface or $\lambda= \pm 1$, then $P$ is loocally flat $(n>1)$.

The is an immediate consequence of the formula

$$
\begin{aligned}
& \bar{R}(X, Y) Z \\
& \quad=R(X, Y) Z+\sigma^{2}\left\{g(Y, Z)\left(\frac{1}{\lambda^{2}} \beta \otimes V+I\right) X-g(Z, X)\left(\frac{1}{\lambda^{2}} \beta \otimes V+I\right) Y\right\}
\end{aligned}
$$

obtained from (4.3) by putting $h=\sigma g$.

Remark. Since in the totally flat case, $\lambda$ is constant, we may consider the one parameter family of hypersurfaces $P_{\lambda}, 0<|\lambda|<1$. For each value of $\lambda, P_{\lambda}$ is a noninvariant hypersurface carrying a globally framed quartic structure $f_{\lambda}$ of rank $2 n-1$, that is,

$$
\begin{array}{cc}
f_{\lambda}^{2}=-I+\tilde{\alpha} \otimes U+\tilde{\beta} \otimes V, \\
f_{\lambda} U=-\lambda V, \quad f_{\lambda} V=0, \\
\tilde{\alpha}(U)=1, \quad \tilde{\alpha}(V)=0, \\
\tilde{\beta}(U)=0, \quad \tilde{\beta}(V)=1 .
\end{array}
$$

$P_{\lambda}$ also carries a globally framed $f$-structure $f^{2}$ of rank $2 n-2$ where

$$
f^{\lambda}=f-\lambda(\tilde{\beta} \otimes U-\tilde{\alpha} \otimes V) .
$$

There are no noninvariant hypersurfaces of a normal contact metric manifold [3].

\section{Metric polynomial manifolds}

An $m$-dimensional $C^{\infty}$ manifold $P$ is said to be pseudo-globally framed (see $\S 2$ ) if there exist a $C^{\infty}$ tensor field $f$ of type $(1,1)$, global vector fields $E_{a}$ and 1 -forms $\eta^{a}, a=0,1, \cdots, 2 \nu$, with $E_{0}=0, \eta^{0}=0$, satisfying

$$
\begin{gathered}
\eta^{a}\left(E_{b}\right)=\delta_{b}^{a}, \\
f^{2}=-I+\left(1-\lambda^{2}\right) \eta^{a} \otimes E_{a}, \quad|\lambda| \leq 1 .
\end{gathered}
$$

If $\lambda=0$, then $\left(f, E_{a}, \eta^{a}\right)$ is globally framed (see [4]). If $\lambda= \pm 1$, then $f$ defines an almost complex structure, so $m$ is even. If, in addition, $m=2 \nu, P$ is a parallelizable almost complex manifold. (Observe that a compact complex parallelizable manifold is Kaehlerian, if and only if, it is a multi-torus [9].) Clearly, the only pseudo-globally framed polynomial structures defined by $f$ are those given by $f^{2}+I=0, f^{3}+f=0$ and $f^{4}+\left(1+\lambda^{2}\right) f^{2}+\lambda^{2} I=0, \lambda \not \equiv \pm 1$, the second case arising by assuming $f E_{a}=0, a=1, \cdots, 2 \nu$, and the latter including $f^{4}+f^{2}=0$ as a special case. In the sequel, we study those structures 
for which $\lambda \neq 0$. The case $\lambda \equiv 0$ was the subject of a previous paper [6].

From (5.2), $f^{2} E_{a}=-\lambda^{2} E_{a}, a=1, \cdots, 2 \nu$.

If $f X=0$, then $X=\left(1-\lambda^{2}\right) \eta^{a}(X) E_{a}$. Applying $f^{2}$ to this relation yields $\lambda^{2}\left(1-\lambda^{2}\right) \eta^{a}(X) E_{a}=0$, so $X=0$ at those points for which $\lambda \neq \pm 1$, since $\lambda \neq 0$. At those $p \in P$ where $\lambda(p)= \pm 1, f_{p}^{2}=-I_{p}$. (We shall presently see that the dimension of $P$ is even.) Hence the linear transformation field $f$ has maximal rank everywhere on $P$.

We assume below that

$$
\begin{gathered}
f E_{2 i-1}=-\lambda E_{2 i}, \\
f E_{2 i}=\lambda E_{2 i-1}, \quad i=1, \cdots, \nu .
\end{gathered}
$$

The pseudo-globally framed manifold $P\left(f, E_{a}, \eta^{a}\right)$ is called a pseudo-globally framed metric manifold if it carries a Riemannian metric $g$ such that

(i) $\eta^{a}=g\left(E_{a}, \cdot\right)$,

(ii) $g(f X, Y)=-g(X, f Y)$.

We put $F(X, Y)=g(f X, Y)$ and call $F$ the fundamental form of $P\left(f, \eta^{a}, g\right)$. From (i) and (ii), we obtain

$$
\begin{aligned}
\eta^{2 i-1} \circ f & =\lambda \eta^{2 i}, \\
\eta^{2 i} \circ f & =-\lambda \eta^{2 i-1} .
\end{aligned}
$$

If we put

$$
J=f-\frac{1}{\lambda} \eta^{2 i-1} \otimes E_{2 i}
$$

then $J$ is an almost complex structure, so $m=2 n$.

Theorem 6. The manifold $P$ with structure tensors $\left(f_{1}, \eta^{a}, g\right), a=1, \ldots, 2 \nu$, where $f_{1}=f-\lambda \eta^{2 i} \otimes E_{2 i-1}, i=1, \cdots, \nu$, is an even dimensional globally framed quartic structure of rank $m-\nu$, that is,

(i) $f_{1}^{2}=-I+\eta^{a} \otimes E_{a}$,

(ii) $\eta^{2 i-1} \circ f_{1}=0, \quad \eta^{2 i} \circ f=-\lambda \eta^{2 i-1}$,

(iii) $f_{1} E_{2 i-1}=-\lambda E_{2 i}, \quad f_{1} E_{2 i}=0$,

(iv) $\eta^{a}\left(E_{b}\right)=\delta_{b}^{a}$,

(v) $f_{1}^{4}+f_{1}^{2}=0$.

Proof. Employing (5.3)-(5.6),

$$
\begin{aligned}
f_{1}^{2} X & =\left(f-\lambda \eta^{2 i} \otimes E_{2 i-1}\right)\left(f X-\lambda \eta^{2 j}(X) E_{2 j-1}\right) \\
& =f^{2} X+\lambda^{2} \eta^{2 j}(X) E_{2 j}+\lambda^{2} \eta^{2 i-1}(X) E_{2 i-1} \\
& =-X+\left(1-\lambda^{2}\right) \eta^{a}(X) E_{a}+\lambda^{2} \eta^{a}(X) E_{a} \\
& =-X+\eta^{a}(X) E_{a} .
\end{aligned}
$$


Moreover, $f_{1} E_{2 i-1}=-\lambda E_{2 i}, f_{1} E_{2 i}=\lambda E_{2 i-1}-\lambda E_{2 i-1}=0$. Hence $f_{1}^{3} X=-f_{1} X$ $-\lambda \eta^{2 i-1}(X) E_{2 i}$, from which $f_{1}^{4} X=-f_{1}^{2} X$.

If $f_{1} X=0$, then $X=\eta^{a}(X) E_{a}$. Applying $f_{1}$ again yields $0=-\lambda \eta^{2 i-1}(X) E_{2 i}$, that is, $\eta^{2 i-1}(X)=0$, so $X=\eta^{2 i}(X) E_{2 i}$.

Theorem 7. The manifold $P$ with structure tensors $\left(f_{2}, \eta^{a}, g\right), a=1, \cdots, 2 \nu$, where $f_{2}=f-\lambda\left(\eta^{2 i} \otimes E_{2 i-1}-\eta^{2 i-1} \otimes E_{2 i}\right)$, is an even dimensional globally framed f-structure of rank $m-2 \nu$, that is,

(i) $f_{2}^{2}=-I+\eta^{a} \otimes E_{a}$,

(ii) $\eta^{a} \circ f_{2}=0, f_{2} E_{a}=0$,

(iii) $\eta^{a}\left(E_{b}\right)=\delta_{b}^{a}$.

Proof. Since $f_{2}=f_{1}+\lambda \eta^{2 i-1} \otimes E_{2 i}, f_{2} E_{2 i-1}=-\lambda E_{2 i}+\lambda E_{2 i}=0$ and $f_{2} E_{2 i}=0$. Hence

$$
\begin{aligned}
f_{2}^{2} X & =\left(f_{1}+\lambda \eta^{2 i-1} \otimes E_{2 i}\right)\left(f_{1} X+\lambda \eta^{2 j-1}(X) E_{2 j}\right) \\
& =f_{1}^{2} X=-X+\eta^{a}(X) E_{a} .
\end{aligned}
$$

Moreover, $f_{2}^{3} X=-f_{2} X+\eta^{a}(X) f_{2} E_{a}=-f_{2} X$.

If $f_{2} X=0$, then $X=\eta^{a}(X) E_{a}$, so rank $f_{2}=m-2 \nu$ since $f_{2} E_{a}=0, a=$ $1, \ldots, 2 \nu$.

A pseudo-globally framed metric manifold is said to be totally flat if the covariant derivatives (with respect to the Riemannian connection) of its structure tensors are zero.

Theorem 8. Let $P_{\lambda}\left(f, \eta^{a}, g\right)$ be a complete totally flat pseudo-globally framed manifold. If $P_{\lambda}$ is simply connected, then there is a Kaehlerian submanifold whose dimension is rank $f$.

Proof. Clearly, $f_{2}$ is also a parallel field, so $D F_{2}$ also vanishes where $F_{2}(X, Y)=g\left(f_{2} X, Y\right)$. Thus $P_{\lambda p}^{\prime}=\left\{X \in P_{\lambda p} \mid F_{2}\left(X, P_{\lambda p}\right)=0\right\}$ defines a parallel distribution, and therefore the orthogonal complement $P_{\lambda p}^{\prime \prime}$ (with respect to $g$ ) also gives a parallel distribution. Observe that the $E_{a}$ do not belong to $P_{\lambda p}^{\prime \prime}$. By the de Rham decomposition theorem $P_{\lambda}=P_{\lambda}^{\prime}+P_{\lambda}^{\prime \prime}$ where $F_{2}$ vanishes on $P_{\lambda}^{\prime}$ and $F_{2}$ has maximal rank on $P_{\lambda}^{\prime \prime}$. Since $D f_{2}$ vanishes so does the Nijenhuis torsion $\left[f_{2}, f_{2}\right]$. The almost complex structure on $P_{\lambda}^{\prime \prime}$ obtained by restricting $f_{2}$ to $P_{\lambda}^{\prime \prime}$ is therefore integrable. $F_{2}^{\prime \prime}$ being closed, $P_{\lambda}^{\prime \prime}$ is symplectic. In fact, since $F_{2}$ has vanishing covariant derivative, $P_{\lambda}^{\prime \prime}$ is a Kaehler manifold.

On a pseudo-globally framed quartic metric manifold, if we define

$$
\bar{f}=f+(1-\lambda)\left[\eta^{2 i} \otimes E_{2 i-1}-\eta^{2 i-1} \otimes E_{2 i}\right]
$$

and

$$
\hat{f}=f-(1+\lambda)\left[\eta^{2 i} \otimes E_{2 i-1}-\eta^{2 i-1} \otimes E_{2 i}\right],
$$

$i=1, \cdots, \nu$, then $\hat{f}$ and $\hat{f}$ are almost complex structures other than $J$. 
Moreover, $(\bar{f}, g)$ is an almost hermitian structure on $P$. Setting $\tilde{F}(X, Y)=$ $g(\bar{f} X, Y)$, we obtain

$$
\tilde{F}=F+2(1-\lambda) \sum_{i} \eta^{2 i} \wedge \eta^{2 i-1}
$$

If the fundamental form $F$ and the $\eta^{a}$ are closed forms, the almost hermitian structure on $P$ is almost Kaehlerian. It is Kaehlerian, if and only if $\bar{f}$ has vanishing covariant derivative with respect to $g$, that is, if $P\left(f, \eta^{a}, g\right)$ is totally flat. In this case, the $E_{a}$ are infinitesimal automorphisms of the Kaehlerian structure. The same conclusion prevails if we consider $\hat{f}$ instead of $\bar{f}$.

Theorem 9. If the pseudo-globally framed metric manifold $P\left(f, \eta^{a}, g\right)$ is totally flat, then it carries the Kaehler structures $(\tilde{f}, g)$ and $(\hat{f}, g)$.

A corresponding theory may be developed for odd dimensional manifolds by letting $a \in\{1, \cdots, 2 \nu+1\}, i \in\{1, \cdots, \nu\}$ and by setting $f E_{2 \nu+1}=0$, in which case from (5.7), $J^{2}=-I+\eta^{2 v+1} \otimes E_{2 v+1}$. Hence $J$ is the fundamental affine collineation of the almost contact manifold $P\left(J, E_{2 v+1}, \eta^{2 \nu+1}\right)$. We give the analogues of Theorems 8 and 9:

Theorem 10. A totally flat odd dimensional pseudo-globally framed metric manifold $P\left(f, \eta^{a}, g\right)$ may be endowed with the cosymplectic structures $(\tilde{f}, \eta, g)$ and $(\hat{f}, \eta, g)$.

Proof. That $P$ is almost cosymplectic is a consequence of the fact that $f$ and the $\eta^{a}$ are covariant constant, and $\lambda=$ const. Thus $\varphi$ and $\eta$ also have vanishing covariant derivatives. The normality of $P$ follows from the vanishing of

$$
\left(D_{\varphi X} \varphi\right) Y-\left(D_{\varphi Y} \varphi\right) X+\varphi\left(D_{Y} \varphi\right) X-\varphi\left(D_{X} \varphi\right) Y+\left\{\left(D_{X} \eta\right)(Y)-\left(D_{Y} \eta\right)(X)\right\} E .
$$

Corollary. Let $P_{2}\left(f, \eta^{a}, g\right)$ be a complete totally flat odd dimensional pseudo-globally framed manifold. If $P_{\lambda}$ is simply connected, then there is a Kaehlerian submanifold of dimension rank $f$.

The proof is similar to that of Theorem 8 .

\section{Normal symplectic structures}

Recall that the framed structure $(f, U, V, \alpha, \beta, \lambda)$ on $P^{\prime}$ is normal if the underlying globally framed $f$-structure $\left(f_{2}, U, V, \tilde{\alpha}, \tilde{\beta}\right)$ is normal. The condition for this is given by the vanishing of the tensor field $\left[f_{2}, f_{2}\right]+d \tilde{\alpha} \otimes U+d \tilde{\beta} \otimes V$ of type $(1,2)$.

The direct product of the pseudo-globally framed hypersurfaces $P_{i}\left(f_{(i)}, U_{i}\right.$, $\left.V_{i}, \tilde{\alpha}_{i}, \tilde{\beta}_{i}, \lambda_{i}\right), i=1,2$, also has the naturally induced almost complex structure $\hat{J}$ on $P_{1}^{\prime} \times P_{2}^{\prime}$ defined by

$$
\begin{aligned}
& \hat{J}_{\left(p_{1}, p_{2}\right)}\left(X_{1}, X_{2}\right)=\left(f_{(1)} X_{1}-\lambda_{1} \tilde{\beta}_{1}\left(X_{1}\right) U_{1}+\lambda_{1} \tilde{\alpha}_{1}\left(X_{1}\right) V_{1}-\tilde{\beta}_{2}\left(X_{2}\right) V_{1}-\tilde{\alpha}_{2}\left(X_{2}\right) U_{1},\right. \\
& \left.f_{(2)} X_{2}-\lambda_{2} \tilde{\beta}_{2}\left(X_{2}\right) U_{2}+\lambda_{2} \tilde{\alpha}_{2}\left(X_{2}\right) V_{2}+\tilde{\beta}_{1}\left(X_{1}\right) V_{2}+\tilde{\alpha}_{1}\left(X_{1}\right) U_{2}\right) \text {. }
\end{aligned}
$$


If the $P_{i}^{\prime}$ are normal, then $\hat{J}$ is integrable [4]. The converse is obtained by employing $[\hat{J}, \hat{J}]\left(X_{1} \times 0, Y_{1} \times 0\right)=0,[\hat{J}, \hat{J}]\left(0 \times X_{2}, 0 \times Y_{2}\right)=0,[\hat{J}, \hat{J}]\left(0 \times X_{2}\right.$, $\left.Y_{1} \times 0\right)=0$ and $[\hat{J}, \hat{J}]\left(X_{1} \times 0,0 \times Y_{2}\right)=0$ in the expression for $[\hat{J}, \hat{J}]\left(X_{1} \times X_{2}\right.$, $\left.Y_{1} \times Y_{2}\right)$ where $X \times Y=(X, Y)$. Define a metric on $P_{1}^{\prime} \times P_{2}^{\prime}$ by $g_{1}+g_{2}$, where $g_{j}=i_{j}^{*} G, j=1,2$, is the metric induced on $P_{j}^{\prime}$ by the almost contact metric $G$ of $M(\varphi, \eta, G)$. Assuming that $M$ is cosymplectic, the 2 -form $\tilde{\Omega}$ on $P_{1}^{\prime} \times P_{2}^{\prime}$ defined by $\tilde{\Omega}=\left(F_{(1)}, 0\right)+\left(0, F_{(2)}\right)+\left(\tilde{\alpha}_{1}, 0\right) \wedge\left(0, \tilde{\alpha}_{2}\right)+\left(\tilde{\beta}_{1}, 0\right) \wedge\left(0, \tilde{\beta}_{2}\right)$, where $F_{(j)}=i_{j}^{*} \Phi, j=1,2$, is the fundamental form of $P_{j}^{\prime}$, is the Kaehler form of $P_{1}^{\prime} \times P_{2}^{\prime}$. For, since $\Phi$ is closed, $F_{(1)}$ and $F_{(2)}$ are closed. The 1 -forms $\tilde{\alpha}_{i}$ and $\tilde{\beta}_{i}, i=1,2$, will also be closed if the $P_{i}^{\prime}$ are totally geodesic. Although the metrics $g_{i}$ of $P_{i}^{\prime}$ need not be Kaehlerian we do have the following result.

Theorem 11. The direct product of the symplectic quartic structures $P_{i}^{\prime}\left(f_{(i)}, U_{i}, V_{i}, \tilde{\alpha}_{i}, \tilde{\beta}_{i}\right), i=1,2$, has a naturally induced almost complex structure given by $\hat{J}$. If the quartic structures are normal, then $\hat{J}$ is integrable, and conversely. If the $P_{i}^{\prime}$ are totally geodesic hypersurfaces and the ambient space is a cosymplectic manifold, then $P_{1}^{\prime} \times P_{2}^{\prime}$ is Kaehlerian.

Corollary. Let $P$ be a totally geodesic hypersurface of a cosymplectic manifold with the induced symplectic quartic structure. Then the direct product of $P$ with itself is Kaehlerian.

Remarks. (a) Let $P\left(f, \eta^{a}, g\right), a=1, \cdots, 2 \nu$, be a totally flat even dimensional pseudo-globally framed manifold. We have seen that $P(\bar{f}, g)$ and $P(\hat{f}, g)$ are Kaehler manifolds. If $P$ is compact, then its topology may be studied from several points of view, first as a compact Kaehler manifold and secondly by introducing a theory on $P\left(f, \eta^{a}, g\right)$ analogous to Weil's generalization of Hodge's theory of harmonic integrals on algebraic varieties. Whereas $\tilde{F}$ and $\hat{F}$ are the Kaehler 2-forms of $P(\hat{f}, g)$ and $P(\hat{f}, g)$ respectively, $F$ plays that role in the latter theory. Although $f$ has maximal rank, $(f, g)$ is not a Kaehler structure on $P$. However, if $\nu=n$, then $(f, g)$ is Kaehlerian. This therefore yields a generalization of Kaehler geometry (see $\S 3$ ).

(b) Let $P\left(f, E_{a}, \eta^{a}, \lambda\right)$ and $\bar{P}\left(\bar{f}, \bar{E}_{a}, \bar{\eta}^{a}, \bar{\lambda}\right)$ be pseudo-globally framed spaces of the same dimension and rank. A diffeomorphism $\mu$ of $P$ onto $\bar{P}$ is called an isomorphism of $P$ onto $\bar{P}$ if

$$
\mu_{*} \circ f=\bar{f} \circ \mu_{*}
$$

and

$$
\mu_{*} E_{a}=\bar{E}_{a}
$$

If $\bar{P}=P$ and $\bar{f}=f, \bar{E}_{a}=E_{a}, \bar{\eta}^{a}=\eta^{a}$ for all $a$, then $\mu$ is an automorphism of $P$. The set of all automorphisms of $P$ clearly forms a group which we denote by $A\left(f, E_{a}, \eta^{a}\right)$. If $\mu \in A\left(f, E_{a}, \eta^{a}\right)$, then $\mu^{*} \eta^{a}=\eta^{a}$, hence $\bar{\lambda} \circ \mu=\lambda$. Moreover, $\mu_{*} \circ f_{2}=f_{2} \circ \mu_{*}$, where $f_{2}=f-\lambda\left(\eta^{2 i} \otimes E_{2 i-1}-\eta^{2 i-1} \otimes E_{2 i}\right)$. Thus $\mu \in A\left(f_{2}, E_{a}, \eta^{a}\right)$, from which we conclude that $\mu \in A\left(\tilde{f}, E_{a}, \eta^{a}\right) \cap A\left(\hat{f}, E_{a}, \eta^{a}\right)$. 
Conversely, if $\mu \in A\left(\bar{f}, E_{a}, \eta^{a}\right) \cap A\left(\hat{f}, E_{a}, \eta^{a}\right)$, then $\mu \in A\left(f, E_{a}, \eta^{a}\right)$. If $P$ is compact and $\left(f, E_{a}, \eta^{a}, \lambda\right)$ is normal, we conclude just as in [5] that the group of automorphisms of a pseudo-globally framed space is a Lie group.

\section{Bibliography}

[1] D. E. Blair, G. D. Ludden \& K. Yano, Induced Structures on submanifolds, to appear.

[2] S. I. Goldberg \& K. Yano, Integrability of almost cosymplectic structures, Pacific J. Math. 31 (1969) 373-382.

[ 3 ] - Noninvariant hypersurfaces of almost contact manifolds, J. Math. Soc. Japan 22 (1970) 25-34.

[ 4 ] _ - Globally framed f-manifolds, to appear in Illinois J. Math.

[ 5 ] _- On normal globally framed f-manifolds, Tôhoku Math. J. 22 (1970) 362370.

[ 6 ] — Polynomial structures on manifolds, Kōdai Math. Sem. Rep. 22 (1970) 199_ 218.

[ 7 ] A. Lichnerowicz, Analytic transformations of Kähler manifolds, Mimeographed notes, University of Illinois, Urbana, Illinois, 1961.

[ 8 ] A. G. Walker, Almost product structures, Proc. Sympos. Pure Math. Vol. 3, Amer. Math. Soc., 1961 94-100.

[9] H. C. Wang, Complex parallisable manifolds, Proc. Amer. Math. Soc. 5 (1954) 771-776.

[10] W. C. Weber \& S. I. Goldberg, Conformal deformations of Riemannian manifolds, Queen's Papers on Pure and Applied Mathematics, No. 16, Queen's University, Kingston, Ontario.

UNIVERSITY OF ILLINOIS 
